\title{
A case of hepatitis B and mycobacterium leprae co-infection causing challenges in diagnosis and treatment
}

\author{
Abate Bane ${ }^{1,2 *}$ \\ ${ }^{1}$ Addis Ababa University, College of health Sciences, Department of Internal Medicine, Addis Ababa, Ethiopia. \\ ${ }^{2}$ Adera medical center, Addis Ababa, Ethiopia.
}

\section{*Corresponding Author: Abate Bane}

Addis Ababa University, College of health Sciences, Department of Internal Medicine, Addis Ababa, Ethiopia. Email: abatebanes@gmail.com

Received: Sep 03, 2021

Accepted: Oct 01, 2021

Published: Oct 08, 2021

Archived: www.jjgastro.com

Copyright: (C) Bane A (2021).

\section{Abstract}

Hepatitis B is a viral infection that attacks the liver and can cause both acute and chronic disease. Most patients are asymptomatic when newly infected. However, some can develop acute hepatitis with symptoms that last several weeks, including yellowing of the skin and eyes, dark urine, extreme fatigue, nausea, vomiting and abdominal pain. There are several extrahepatic manifestations that are associated with chronic HBV infection, including arthralgia, weakness, nephritis, and generalized vasculitis.

Leprosy is also an infectious disease caused by a bacillus, Mycobacterium leprae, which multiplies slowly. Leprosy mainly affects the skin, the peripheral nerves, mucosa of the upper respiratory tract, and the eyes.

The co-infection of HBV and mycobacterium leprae can lead to various manifestations and complications. Hepatitis B co-infection worsen course of leprosy. Leprosy treatment can also lead to flare of latent HBV infection.

In this case report, we discuss the challenges faced in diagnosing and treating co-infection and complication of leprosy in a patient with chronic hepatitis $B$ infection.

Keywords: leprosy; hepatitis B; leprosy reaction; vasculitis.

\section{Introduction}

Hepatitis B is a DNA hepatotropic virus that attacks the liver and can cause both acute and chronic liver disease. The virus is most commonly transmitted from mother to child during delivery, as well as through contact with contaminated blood or other body fluids, including sex with an infected partner, injection-drug use that involves sharing needles, syringes, or drug-preparation equipment, and needle sticks or exposures to sharp instruments. Fortunately, hepatitis B can be prevented by vaccines that are safe and effective.
Most people (90\%) do not experience any symptoms when newly infected. However, some develop acute illness with symptoms that last several weeks, including yellowing of the skin and eyes, dark urine, extreme fatigue, nausea, vomiting and abdominal pain. A small subset of patients with acute hepatitis can develop acute hepatic failure, which can lead to death. In $5-10 \%$ of adults, hepatitis B viral infection can progress to chronic hepatitis that can later evolve into cirrhosis and hepatocellular cancer [1]. 
Citation: Bane A. A case of hepatitis B and mycobacterium leprae co-infection causing challenges in diagnosis and treatment. Japanese J Gastroenterol Res. 2021; 1(5): 1022.

Hepatitis B infection can also cause several immune mediated extrahepatic manifestations that are associated with chronic HBV infection, causing significant morbidity and mortality. These include purpura, arthralgia, weakness, nephritis, pulmonary disease, and generalized vasculitis [2].

Leprosy is caused by a bacillus, Mycobacterium leprae, which multiplies slowly with incubation period ranging from 1-5 years or even more, and mainly affects the skin, the peripheral nerves, mucosa of the upper respiratory tract, and the eyes. The bacillus is likely transmitted via droplets, from the nose and mouth, during close and frequent contact with untreated cases. Even though, leprosy is curable with multidrug therapy (MDT), it can cause progressive and permanent damage to the skin, nerves, limbs, and eyes, if untreated [3].

Sudden changes in immune-mediated response to mycobacterium leprae antigen lead to leprosy reactions. Type 1 leprosy reaction is also called reversal reaction, false exacerbation reaction or upgrading reaction and is reversible, while, type 2 subtype also called downgrading reaction; is associated with disease worsening. Upgrading (reversal) reactions occur in patients receiving anti-leprosy therapy, while, downgrading reactions occurs in patients who do not receive therapy [4].

Reversal reaction episodes often occur within the first 6 months of initiating multidrug therapy (MDT), manifesting as erythematous and edematous skin lesions which later become scaled and rarely ulcerated. The lesions are often painful and tender accompanied by edema and neuritis in the face and extremities.

The risk of type 1 reaction may increase with vaccination, MDT, pregnancy, puerperality, infections, stress, trauma, and oral contraceptive use. The extensiveness of leprosy skin lesions has also been described as an important risk factor both in patients with low and high bacilli load [4].

A cohort Study conducted on viral co-infection and leprosy showed that HBV co-infection was associated with a higher rate of leprosy related neuritis and nerve function impairment. HBV and/ or HCV co-infection with leprosy noted to precipitate leprosy reaction as well [5].

\section{Case presentation}

TT (MRN-2933) is a 28 year old male patient; an unmarried banker from Muger town in West Shewa, Ethiopia. He first presented to Adera medical center on September 25, 2009 with a complaint of headache and anorexia, but had no remarkable sign. His investigation showed normal complete blood count, renal function test, abdominal ultrasound and HCV test results, while, aspartate aminotransferase and alanine aminotransferase were elevated 2 and 4 times the upper normal limit, respectively. He also tested positive for hepatitis B surface antigen, for which he was started on tenofovir.

His symptoms resolved after he started treatment and his liver function test results also normalized, while, his hepatitis $B$ e-antigen and antibody were both positive. However, he discontinued tenofovir after 1 year and 7 months as he felt cured when his symptoms disappeared.
Unfortunately, he returned to Adera Medical Center with complaints of anorexia, abdominal pain, and yellowish urine after 5 months of tenofovir discontinuation. He was then investigated again with complete blood count, liver function test, renal function test, hepatitis B viral load and abdominal ultrasound examination, of these all were normal except for HBV viral load, which was $50 \mathrm{lU} / \mathrm{ml}$. He was then re-started on tenofovir and continued his follow up. HBV viral load dropped to less than 20 $\mathrm{IU} / \mathrm{ml}$ after six months of reinitiating tenofovir.

However, six years after he started tenofovir for chronic hepatitis B infection, he developed patchy body aches, polyarthralgia, painful feet swelling, anorexia, fatigue, and insomnia for which he was treated with prednisolone with a diagnosis of vasculitis which resolved within a month.

But he returned again after three months complaining of ankle swelling, polyarthralgia, and papular skin rashes over hands and face, while, taking tenofovir and tapered dose of prednisolone (Figure 1 and 2). However, there was no nerve enlargement noted. For these, he was referred to a dermatologist and diagnosed to have multi-bacillary leprosy after a skin snip test (Figure 3) and was started on anti- leprosy medication including dapson, rifampicin and clofazimine (MDT).

Eleven days after he started the MDT, he developed nodular lesions over the hands and face, difficulty of moving his limbs with polyarthralgia. He also had post pruritic eruption and crusted lesion at the right elbow joint. He was diagnosed to have leprosy reaction (type 1 reaction) and was admitted to ward for one week where he was started on IV hydrocortisone while he continued taking TDF and MDT (dapson, rifamcin and clofazimine) and discharged improved and continued his MDT and TDF with appointment for regular follow up care.

He had no history of allergy, diabetes, hypertension, liver disease, contact with a person having jaundice or skin lesion, or a family history of leprosy.

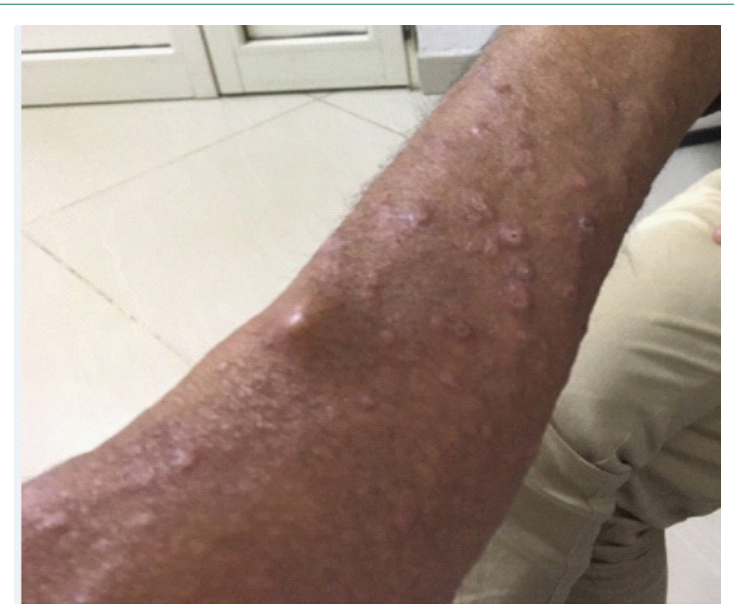

Figure 1: Photograph of papular skin lesions on the patient's right hand, Adera Medical Center, November, 2018. 


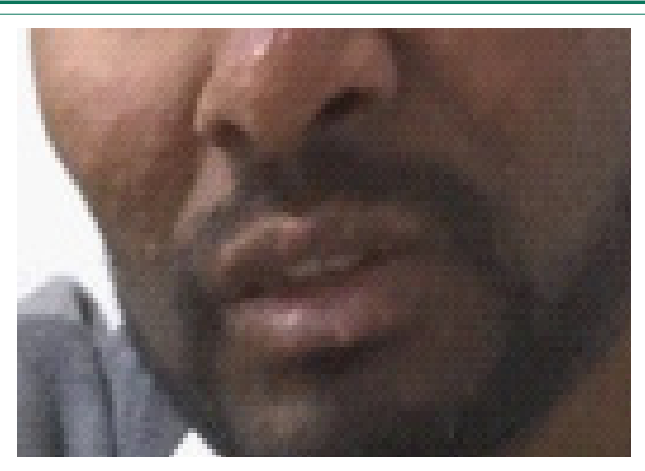

Figure 2: Photograph of papular skin lesions on the patient's face, Adera Medical Center, November, 2018.

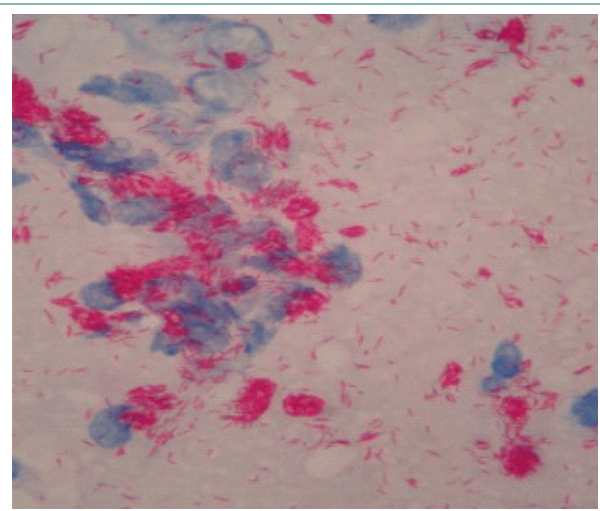

Figure 3: Microscopic picture of Mycobactrium laprae from the patient's skin snip, Adera Medical Center, November, 2018.

\section{Discussion}

Our patient had HBV and leprosy co-infection and later developed type 1 leprosy reaction, which was likely precipitated by HBV co-infection. Leprosy reaction was initially misdiagnosed as it mimics extrahepatic HBV vasculitis.

There have been reports and studies on the interaction of these co- infections. A study conducted in 1977 in South Africa on hepatitis $B$ antigen and hepatitis $B$ antibody positivity on different subtypes of leprosy and controls showed that there was no statistical difference in the incidence of $\mathrm{HBsAg}$ and $\mathrm{HBsAb}$ positivity among the three groups or between normal controls and the leprosy patients [6]. Another study conducted in 1983, in India, on occurrence of hepatitis B virus infection among patients with leprosy also concluded that there was no association between carrier-ship of HBsAg and lepromatous leprosy [7].

However, a study done in 1978 among Melanesian people concluded that lepromatous patient have an Impaired Immune response that not only predisposes them to the most severe form of leprosy but may also decrease their efficiency in clearing HBsAg infection with anti-HBs antibodies [8].

Also another study, conducted in 2003 , in India on hepatic involvement and hepatitis B surface antigenemia in leprosy showed HbsAg was detected in $7.54 \%$ of lepromatous leprosy patients as compared to $2 \%$ of the normal healthy controls [9].

A more recent study that took place in Brazil in 2013 also revealed leprosy patients are susceptible to develop hepatitis $B$ virus infection. Institutionalized patients, who probably present a stronger Th2 response, have higher risk of being exposed to hepatitis $B$ virus. This clearly emphasizes the need for special care to leprosy patients in preventing hepatitis B virus co-infection [10].
Our patient's presentation was not typical for leprosy, thus his symptoms were initially attributed to vasculitis as extra hepatic manifestations of chronic hepatitis $B$. And had it not been for a high index of suspicion, the diagnosis of leprosy would have been delayed or missed.

As infections are among risk factors for the development of leprosy reaction, it is likely that chronic hepatitis B infection is the precipitant to leprosy reaction in our patient [6]. A study done in Brazil, in 2007 suggested that hepatitis B and hepatitis $C$ infections may have a role in the development of leprosy reversal reactions, thus could be considered as risk factors for reversal reaction [11].

In conclusion, due to immune dysregulation, HBV co-infection is common and can precipitate reaction among leprosy patients. Hence, having high index of suspicion of leprosy co infection and leprosy reaction when HBV patients present with unexplained vasculitis syndrome is essential for early diagnosis and treatment. Likewise, testing leprosy patients for viral hepatitis is also necessary especially for those presenting with leprosy reaction.

Acknowledgements: We are grateful to the patient and all our staff involved in the care of this patient.

\section{References}

\section{WHO, Hepatitis B, 27 July 2021}

2. Shim, Michael, and Steven-Huy Han. "Extrahepatic manifestations of chronic hepatitis B." Hepatitis B Annual. 2006; 3: 1.

3. Leprosy (Hansen's disease), WHO. 2021.

4. Bilik, Leyla \& Demir, Betul \& Cicek, Demet. Leprosy Reactions. Hansen's Disease - The Forgotten and Neglected Disease. 2019.

5. Machado, Paulo \& Machado, Lídia \& Shibuya, Mayume \& Rego, Jamile \& Johnson, Warren \& Glesby, Marshall. Viral Co-infection and Leprosy Outcomes: A Cohort Study. PLoS neglected tropical diseases. 2015; 9: e0003865.

6. Sher R, Mackay ME, Macnab GM, Kok SH, Koornhof HJ. Hepatitis B Antigen, Hepatitis B Antibody, and Subtypes in Leprosy, Infect Immun. 1977.

7. Katoch V, Kiran K, Dutta A, Sharma, V, Ramu G. Hepatitis-B virus infection in patients with leprosy - A brief communication. Leprosy in India. 1983; 55: 193-6.

8. Serjeantson S, Graeme W. Immune response of leprosy patients to hepatitis B. American journal of epidemiology. 1978; 107: 321-7.

9. Pramod N, Gupta G, Khare A. Hepatic involvement and hepatitis B surface antigen ( $\mathrm{Hbs} \mathrm{Ag}$ ) in leprosy. Indian journal of dermatology, venereology and leprology. 2003; 69: 32-4.

10. Cleverson L, Denis U, Anna Carolina B, Angelica B, lara R. Leprosy and hepatitis $B$ coinfection in southern Brazil. The Brazilian journal of infectious diseases: An official publication of the Brazilian Society of Infectious Diseases. 2013; 18.

11. Rego VP, Machado PR, Martins I, Trindade R, Paraná R. Type 1 reaction in leprosy: Characteristics and association with hepatitis B and C viruses. Rev Soc Bras Med Trop. 2007; 40: 546-9.

12. José $R$, Ágabo $S$, Regina M. Prevalence of hepatitis $B$ and $C$ virus infection among leprosy patients in a leprosy-endemic region of central Brazil. Memórias do Instituto Oswaldo Cruz. 2011; 106 : $632-4$. 